\title{
Risiko Impfen?
}

\section{Warum Menschen sich und ihre Kinder nicht impfen lassen}

Frank Kowalzik, Fred Zepp

\section{Grundsätzlich sind die Erfolge von Impfungen und Impfprogrammen für die Prävention von Infektionskrankheiten und deren Komplikationen weltweit anerkannt. Dennoch fällt aktuell gerade in industrialisierten Staaten ein Vertrauensschwund auf: Impfungen und öffentlich empfohlene Impfprogramme werden immer weniger wahrgenommen. Stich- wort „Impfskepsis“. Die WHO sieht darin mittlerweile ein komplexes globales Problem. Doch wie ist es lösbar?}

\section{DEFINITION}

Unter Impfskepsis („vaccine hesitancy“) versteht die Weltgesundheitsorganisation (WHO) eine verzögerte Akzeptanz von Impfungen bis hin zur vollständigen Ablehnung von Impfprogrammen.

Für die steigende Impfskepsis lässt sich eine Vielzahl von Gründen anführen, die im Folgenden diskutiert werden. Dabei steht immer auch die Sicherheit neuer Impfstoffe im Fokus. Die Zahl wissenschaftlicher Publikationen, die sich mit der Impfstoffsicherheit befassen, illustriert dies eindrucksvoll ( $\triangleright$ Abb. 1). Gab es 1998 gerade einmal 200 Publikationen pro Jahr, sind es heute durchschnittlich ungefähr 6-mal so viele.

\section{ZUSATZINFO}

\section{Folgen des Nicht-Impfens}

Die abnehmende Bereitschaft, anerkannt wirksame Präventionskonzepte wie Impfstoffe konsequent zu nutzen, hat gravierende Folgen. Umfangreiche Studien [1] belegen: Im Vergleich zu geimpften Kindern haben die Kinder, deren Eltern

- Pertussis-Impfstoffe ablehnen, eine 23-mal höhere Wahrscheinlichkeit, Keuchhusten zu bekommen;

- eine Varizellen-Impfung ablehnen, eine 9-mal höhere Wahrscheinlichkeit für eine Varizella Zoster-Infektion;

- Pneumokokken-Konjugat-Impfstoffe ablehnen, eine 6-fach erhöhte Wahrscheinlichkeit für eine invasive Pneumokokkenerkrankung oder Lobärpneumonie.

Manche Staaten und Regionen gewähren großzügig Ausnahmen von der Teilnahme an Impfprogrammen. Studien zeigen, dass dort häufiger impfpräventable Krankheiten wie Masern, Mumps oder Keuch- husten auftreten [2]. Ablehnen oder verzögertes Durchführen von Impfungen trägt entscheidend zur Morbidität und Letalität von grundsätzlich impfpräventablen Infektionskrankheiten bei.

\section{Hintergrund}

Skepsis gegenüber Impfstoffen ist kein neues Phänomen: Seit dem 18. Jahrhundert wachsen bei jeder Einführung eines neuen Impfstoffs Angst und Misstrauen in der Bevölkerung [3]. Selbst zu Zeiten der oft tödlich verlaufenden Pockenepidemien gab es einen öffentlichen Widerstand gegen den neuen Pockenimpfstoff. Großbritannien führte daraufhin Pflichtimpfungen ein. In den USA musste 1905 die Pflichtimpfung gegen Pocken sogar vor dem Obersten Gerichtshof verhandelt werden („Jacobson gegen Massachusetts“). In letzter Instanz bestätigte das Gericht damals das Recht einzelner Bundesstaaten, diese Pflichtimpfungen durchzusetzen.

\section{Merke}

Häufig wird das Verschwinden der durch Impfungen verhinderten Infektionskrankheiten als Ursache für die abnehmende Impfbereitschaft angeführt - Impfstoffe sind gewissermaßen Opfer ihres eigenen Erfolgs geworden.

Die erfolgreiche Prävention schwerer, früher oft tödlich verlaufender Infektionen durch Impfungen hat tatsächlich die Bedrohung durch Erkrankungen und damit verbundene Risiken aus der Wahrnehmung der Menschen verschwinden lassen. Wenn aber die Bedrohung nicht mehr präsent ist, rückt der Fokus des öffentlichen Interesses auf das vermeintliche Impfrisiko. Vermutete Impfnebenwirkungen werden als größere Gefahr wahrgenommen, selbst wenn sie 1000-fach seltener auftreten als die Komplikationen von Infektionskrankheiten. 


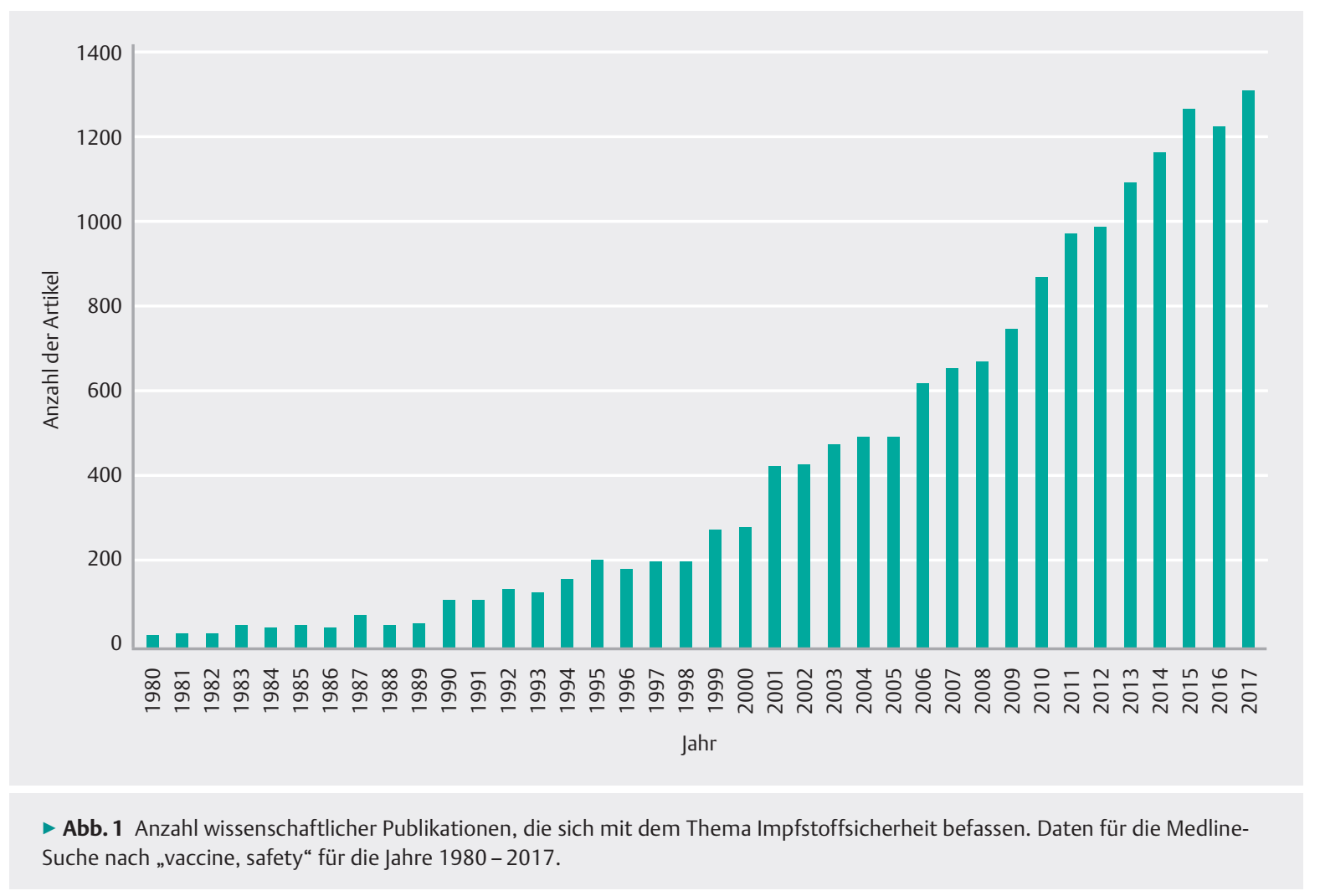

Mit der Entwicklung zunehmend komplexer, teilweise gentechnologisch hergestellter Impfstoffe ist die Sachlage für medizinische Laien und auch Mediziner noch unübersichtlicher geworden. Bei immer schwieriger vermittelbarem Wissen über moderne Impfstoffe sind vermutete impfstoffassoziierte Risiken für Laien und Fachleute häufig nicht mehr rational einschätzbar. Wie also kann man das Ziel erreichen, auch zukünftig die optimale Nutzung von Impfstoffen in der Bevölkerung sicherzustellen? Dazu muss man in einem ersten Schritt die öffentliche Wahrnehmung von Impfungen und die damit verbundenen Mechanismen individueller Entscheidungsprozesse verstehen. Der zweite Schritt wäre dann, wirksame Kommunikationskonzepte zu entwickeln. Beide Schritte beleuchtet dieser Beitrag.

\section{Öffentliche Wahrnehmung}

\section{VCP-Bericht 2015}

Seit 2010 untersucht das Vaccine Confidence Project (VCP) in London die öffentliche Wahrnehmung von Impfungen. Um die Fragestellung wissenschaftlich strukturiert zu bearbeiten, wurde der Vaccine Confidence Index ( $\mathrm{VCl}$, Vertrauensindex in Impfungen) entwickelt. Er umfasst einen Fragenkatalog, anhand dessen vergleichbare Daten zum Wissen um und zum Vertrauen in Impfungen erhoben werden können [4]. Das Modell wurde 2014 erstmals in Nigeria, Pakistan, Indien, Georgien und
Großbritannien eingesetzt, die Ergebnisse wurden im VCP-Bericht 2015 publiziert. Die Befragung von mehr als 20000 Personen ergab u.a., dass in Großbritannien Eltern von Kindern unter 5 Jahren am stärksten zögern, ihre Kinder zeit- und empfehlungsgerecht impfen zu lassen. Vermuteter Grund: In Großbritannien scheint das Vertrauen in Impfprogramme grundsätzlich eng mit dem Vertrauen in das staatliche Gesundheitswesen zu korrelieren. In Nigeria, Pakistan und Indien hingegen vertrauen Menschen öffentlichen Impfprogrammen stärker als den jeweiligen landestypischen Gesundheitssystemen.

\section{VCP-Bericht 2016}

Der VCP-Bericht aus dem Jahr 2016 präsentiert UmfrageDaten aus insgesamt 68 Ländern, darunter auch Deutschland [5]. Zwischen September und Dezember 2015 wurde weltweit 65819 Personen (pro Land jeweils etwa 500 Frauen und 500 Männern) in persönlichen Interviews, Telefonsurveys oder per Online-Erhebungen der Fragenkatalog des VCl vorgelegt. Die Erhebung zeigt zunächst: Impfungen werden von der Weltbevölkerung überwiegend als wichtig wahrgenommen. Regional ergeben sich jedoch deutliche Unterschiede. So sind in Europa Impfentscheidungen eher von Skepsis und eingeschränktem Vertrauen in die Sicherheit von Impfstoffen geprägt, während in den Ländern des Westpazifiks und in Südostasien eher religiöse Kriterien relevant sind. 


\section{Vertrauen}

In der WHO-Region Europa finden sich 7 der 10 Länder mit dem geringsten Vertrauen in die Sicherheit von Impfungen. Ein Maß für das Vertrauen in Impfungen ist die Haltung zur Aussage: „Insgesamt glaube ich, dass Impfungen sicher sind" ("overall I think vaccines are safe”). Mit „eher nicht“ oder sogar „keinesfalls“ urteilten

- in Frankreich 41 \% der Befragten (siehe Infobox),

- in Bosnien-Herzegowina 36\%,

- in Russland $28 \%$,

- in der Mongolei $27 \%$ und

- in Griechenland, der Ukraine und Japan jeweils $25 \%$.

Im weltweiten Durchschnitt waren dagegen nur $12 \%$ der Befragten skeptisch gegenüber Impfstoffen eingestellt.

\section{Merke}

Die Stichprobe aus Deutschland ergab bei 10,5\% der Befragten Zweifel an der Sicherheit von Impfstoffen. Der Aussage „Impfungen sind wichtig für Kinder“ stimmten 92,2\% aller Befragten in Deutschland zu.

\section{ZUSATZINFO \\ Frankreich}

Es ist beachtenswert, dass Frankreich trotz eines sehr gut funktionierenden, zentralistisch strukturierten Gesundheitssystems in der Auswertung des VCP das Land mit dem geringsten Vertrauen in Impfstoffe war. Eine aktuelle Telefonbefragung dort bestätigt dies [6]:

- $46 \%$ der befragten Eltern gaben eine skeptische oder ablehnende Haltung gegenüber Impfungen gegen Masern und Hepatitis B (HBV) an,

- 48 \% gegenüber der Humanen Papilloma Virus (HPV) -Impfung von Mädchen und

- 35 \% der älteren Befragten gegenüber einer Grippeimpfung.

Die negative Haltung gegenüber Impfungen war ausgeprägter bei Menschen mit höherem Bildungsniveau und bei älteren Menschen mit negativer Einschätzung des eigenen Gesundheitszustandes. Eine skeptische bzw. ablehnende Haltung zu allen vier in der Studie untersuchten Impfungen (Masern, HBV, HPV, Influenza) war stark assoziiert mit einer ungünstigen Wahrnehmung des Nutzen-Risiko-Verhältnisses oder/und mit Unsicherheit darüber.

\section{Religion}

Wie schon in der ersten Erhebung, sehen auch im aktualisierten VCP-Bericht Befragte aus der WHO-Region Südostasien (Bangladesch, Indonesien, Thailand und Indien) Impfungen überwiegend als sicher an. In den WHO-Regionen Südostasien und Westlicher Pazifik befürchten die Befragten am häufigsten, dass Impfen mit ihren religiösen Überzeugungen in Konflikt geraten könne (25,7 bzw. 24,3\%). Diese Bedenken sind besonders stark ausgeprägt in der Mongolei (50,5\%), Thailand $(44,4 \%)$ und in Vietnam (31,8\%). Im weltweiten Durchschnitt geben 15,4\% aller Befragten religiöse oder soziokulturelle Überzeugungen als möglichen Grund für eine zögerliche oder ablehnende Haltung gegenüber Impfungen an; in der Stichprobe aus Deutschland sind es 8,9\%.

\section{Weitere Studien}

\section{Politische Einstellung und Vertrauen}

Eine internetbasierte Studie [7] in den USA befasste sich mit der Frage: In welcher Weise beeinflussen a) politische Überzeugungen und b) Vertrauen in medizinisches Personal und Gesundheitsbehörden die Einstellung zu Impfungen gegen Influenza, Pertussis und Masern? (Anmerkung: politische Einstellung beschreibt im Kontext der Studie Grundvorstellungen der Befragten über die Struktur und Ordnung der Gesellschaft.) Ergebnisse:

- Politisch konservative Teilnehmer neigten eher dazu, Impfungen negativ zu bewerten.

- Sehr konservativ eingestellte Personen äußerten ein geringeres Vertrauen in Einrichtungen wie das Center for Disease Control (CDC) als weniger Konservative.

- Die politische Überzeugung schien hingegen keinen Einfluss auf das Vertrauen gegenüber Kinder- und Familienärzten zu haben.

- Personen mit grundsätzlich hohem Vertrauen in medizinische Leistungserbringer (wie Familien- und Kinderärzte oder Experten der Gesundheitsbehörden) zeigten eine deutlich höhere Impfbereitschaft.

- Ältere Menschen hatten eine tendenziell negativere Einstellung zum Impfen.

- Ein gutes Einkommen wirkte sich grundsätzlich positiv auf die Impfbereitschaft aus.

\section{Andere Faktoren}

- In Griechenland [8] waren sozioökonomische Faktoren wie die Zahl von Geschwistern und die Ausbildung des Vaters die wichtigsten prädiktiven Faktoren für Impfakzeptanz und Impfbereitschaft.

- Eine Studie in Nigeria ergab: An unvollständigen Immunisierungen waren eher die Mütter „schuld“ durch unzureichende Impfkenntnisse, während NichtImmunisierungen durch die ablehnende Haltung der Väter bedingt war [9].

- Eine weitere Studie zur MMR-Impfung in Großbritannien zeigte, dass mehrere Faktoren die Entscheidungsfindung sogar bei jeder einzelnen Impfdosis beeinflussen können. Dabei variierte auch die Stärke des Einflussgrades bei jeder Dosis [10]. 
Fazit

Grundsätzlich ist festzustellen: die Gründe, ob eine Person sich für oder gegen Impfungen entscheidet, sind außerordentlich vielfältig. Auch wenn zunehmend Faktoren bekannt werden, die die mangelnde Impfbereitschaft in verschiedenen Populationen beeinflussen [11 - 14], so sind noch lange nicht alle relevanten Einflussgrößen identifiziert $[10,15]$. Insofern wird man kaum eine universelle Strategie für eine bessere Akzeptanz von Impfungen entwickeln können. Allerdings kristallisiert sich in nahezu allen Erhebungen der Faktor „Vertrauen in Impfstoffe und/oder das Gesundheitssystem “ als essenziell für eine positive Impfentscheidung heraus. Das Vertrauen in Impfungen korreliert wiederum mit der Einschätzung a) des speziellen individuellen Erkrankungsrisikos und b) der potenziellen Nebenwirkungen der Impfstoffe.

\section{Risikowahrnehmung und Impfent- scheidungen}

Die Strategic Advisory Group of Experts (SAGE) der WHO hat drei Kriterien als Einflussfaktoren für Impfskepsis identifiziert:

- Confidence (Vertrauen)

- Complacency (Gleichgültigkeit)

- Convenience (Verfügbarkeit)

\section{Merke}

Während die Verfügbarkeit von Impfstoffen in wohlhabenden industrialisierten Staaten kaum ein Problem ist, sind hier mangelndes Vertrauen und Indifferenz gegenüber Gesundheitsrisiken durch Infektionen relevant für das Impfverhalten.

"Confidence“ ist in vielerlei Hinsicht erforderlich: Vertrauen in die Wirksamkeit und Sicherheit von Impfungen, Vertrauen in das Gesundheitssystem generell und Vertrauen in die Motive der Entscheidungsträger. Hohes Vertrauen geht einher mit

- einer positiven Impfeinstellung,

- besserem Wissen über Impfungen und

- einer höheren Impfbereitschaft.

Menschen mit geringem Vertrauen besitzen hingegen häufig nur begrenzte Kenntnisse über Impfstoffe, zweifeln an deren Sicherheit und den Aussagen medizinischer Experten und neigen hinsichtlich der Interessen staatlicher Institutionen zu verschwörungstheoretischem Denken [16-18].

Grundlage einer erfolgreichen Prävention von Infektionen sind die Impfprogramme für Kinder und Jugendliche. Naturgemäß kommt hier den Eltern die entscheidende Rolle zu:
- Zweifeln Eltern an der Sicherheit von Impfstoffen, trägt dies wesentlich zu verminderten Durchimpfungsraten bei.

- Darüber hinaus betreffen elterliche Bedenken die häufigen Injektionen oder die Frage, wie viele Impfstoffe das Immunsystem von Säuglingen oder jungen Kindern überhaupt sicher verarbeiten kann.

Daraus entwickelt sich nicht selten ein starkes Gefühl von Unsicherheit und Ohnmacht, das die individuelle Risikoabwägung für oder gegen eine Impfung zuweilen ins Metaphysische verlagert. Auch wird es zunehmend schwieriger, neue Impfstoffentwicklungen gut verständlich in der Öffentlichkeit zu kommunizieren. Viele dieser neuen Präparate sind komplexe medizinische Produkte, die neben Impfantigenen neuartige Zusatzstoffe wie immunstimulatorische Adjuvanzien enthalten. Nicht nur für medizinische Laien sind die wissenschaftlichen Grundlagen von Impfstoffkomponenten und Zusatzstoffen schwer nachzuvollziehen.

\section{DEFINITION}

Risikowahrnehmung und Risikoabschätzung bestimmen wesentlich die individuelle Impfentscheidung. „Risikowahrnehmung“ bedeutet: die Bewertung des Risikos einer potenziellen Gefahrenquelle durch Laien. Der Begriff wird in der Risikoforschung verwendet, um die subjektive Risikowahrnehmung von Laien gegenüber der objektiven Risikoabschätzung durch Experten abzugrenzen [19].

„Allgemeine Risikowahrnehmung“ beschreibt generelle Vorstellungen von Gesundheitsrisiken (z. B. „Rauchen und Alkohol schaden der Gesundheit“). „Selbstbezogene Risikowahrnehmung“ beschreibt die Einschätzung der persönlichen Gesundheitsbedrohung.

\section{Was beeinflusst die Risikowahrnehmung?}

\section{Defensiver Optimismus}

Externe und interne Faktoren beeinflussen, wie wir ein Risiko wahrnehmen. Das heißt: objektive Risikoinformationen werden individuell unterschiedlich bewertet. Grundsätzlich beurteilen Menschen Aktivitäten oder Gefahrenquellen, die sie persönlich kennen und denen sie sich freiwillig aussetzen, als weniger bedrohlich und riskant als solche, die sie nicht kennen und/oder über die sie keine direkte Kontrolle haben. So können Personen gut über ein Risiko informiert sein, sich aber selbst für weniger gefährdet halten. Beispielsweise wird mit zunehmendem Alter das eigene Risiko für einen Herzinfarkt höher eingeschätzt. Gleichzeitig bewertet man jedoch in der Regel das eigene Risiko geringer als bei einer anderen 
Person gleichen Alters und Geschlechts - so bleibt eine für die eigene Person vorteilhafte Differenz bestehen.

Merke

Dieses Verhalten wird als „defensiver Optimismus“ bezeichnet: Das Individuum neigt dazu, die Realität selbstwertdienlich zu verzerren.

Infolge derartiger Verzerrungen bei sozialen Vergleichsprozessen erscheint das eigene Erkrankungsrisiko (, komparatives Risiko") häufig geringer als das entsprechende Erkrankungsrisiko anderer Menschen - obwohl es keine objektiven Unterschiede gibt [18].

\section{Seltene vs. häufige Risikofaktoren}

Ein weiterer Aspekt ist die Bewertung der persönlichen Kontrollierbarkeit eines Risikos bzw. des Risikoverhaltens. Seltene Risikofaktoren nimmt man, wenn sie denn auftreten, in ihrer Bedeutung stärker wahr als häufiger vorkommende Risikofaktoren. Schon eine frühe Studie [20] zeigte: Dramatische Todesursachen (Unfälle, Mord) werden entgegen der Realität in ihrer Eintrittshäufigkeit überschätzt und weniger dramatische (Infektionserkrankungen) unterschätzt. Dieses Verhaltensmuster begünstigt eine nachlässige oder auch gleichgültige Einstellung („complacency“) gegenüber impfpräventablen Infektionen. Andererseits ist bekannt: wenn plötzlich das Aufkommen von Erkrankungen im Umfeld einer Person steigt (z. B. bei eigentlich sehr seltenen Meningitisfällen), schätzt sie recht schnell das individuelle Erkrankungsrisiko höher ein. Die subjektive Risikowahrnehmung entspricht also selten den objektiv messbaren Fakten - vielmehr wird sie durch die individuelle Wahrnehmung in beide Richtungen (Unter- und Überschätzung) verzerrt.

\section{Gefahrenspezifische Risikowahrnehmung}

Eine Metaanalyse zeigt: Eine zunehmende gefahrenspezifische Risikowahrnehmung hat einen Haupteffekt auf das Impfverhalten Erwachsener [21]. In der Risikobewertung besteht in der Regel ein Gleichgewicht zwischen wahrgenommenem Risiko und Nutzen von Impfstoffen. Wenn Bedenken für eine durch Impfung vermeidbare Krankheit zunehmen (z. B. im zeitlichen Zusammenhang mit einem Ausbruch), verbessert sich in der Regel sehr schnell auch die Impfakzeptanz. Fällt hingegen die Rate an schwerwiegenden Infektionen infolge der erfolgreichen Impfprävention, steigt die Zahl derjenigen, die den Impfstoff mehr fürchten als die Krankheit, die er verhindert.

\section{Öffentlichkeit}

Masern und Autismus

Öffentlichkeitswirksame Berichte über angebliche schwerwiegende Impfstoffnebenwirkungen haben dem Impfwesen immer wieder enormen Schaden zugefügt. Das wohl bekannteste Beispiel ist eine Publikation des
Gastroenterologen Andrew Wakefield in „The Lancet“, in dem der Autor Daten verfälschte, um eine kausale Verbindung zwischen MMR-Impfstoff und Autismus herzustellen. Obwohl die Aussagen später wissenschaftlich widerlegt [22] und der Artikel zurückgezogen wurden, war der Imageschaden für die Masern-Impfung verheerend. Insbesondere durch Einbinden von Laienpresse und Internet war es Wakefield gelungen, eine große, aktive Impfgegnerschaft zu mobilisieren und so zu einer erheblichen Verunsicherung der Bevölkerung beizutragen. Infolge der über Monate anhaltenden Diskussionen um Wakefields Aussagen fielen in England die Masern-Impfraten deutlich ab, gefolgt von einer signifikanten Zunahme von Wild-Masern-Infektionen. Obwohl ihm in England die medizinische Lizenz entzogen wurde, ist Andrew Wakefield in den Vereinigten Staaten als Impfgegner aktiv. In 2017 inszenierte er sogar einen Dokumentarfilm gegen Impfstoffe („Vaxxed: Von der Vertuschung zur Katastrophe“), in dem er unverändert seine falschen Aussagen und Hypothesen verbreitet.

\section{Komödie mit „Information“}

Auch durchaus gut gemeinte Versuche, das Thema Impfskepsis in den Laienmedien zu bearbeiten, können in der öffentlichen Wahrnehmung die Impfbereitschaft beeinträchtigen. „Eingeimpft - Familie mit Nebenwirkungen“ lautet der Titel einer aktuellen Dokumentation, in der der Filmemacher David Sieveking sich mit dem Thema Impfen auseinandersetzt. Der vom Regisseur als Beziehungskomödie bezeichnete Film ist autobiografisch angelegt und zeigt, wie der Filmemacher bei der Geburt seiner ersten Tochter mit seiner Freundin versucht, eine individuelle Impfentscheidung zu treffen. In der Fachwelt erntet der Beitrag unisono Kritik. Mit dem Anspruch, umfassend alle Meinungen zum Impfen darlegen zu wollen, stellt der Regisseur unkritisch widersprüchliche Szenen und Positionen - teilweise auch Fehlinformationen und wissenschaftlich widerlegte Hypothesen - nebeneinander, ohne diese hinsichtlich ihrer Seriosität einzuordnen. Ohne eine fachliche Bewertung der dargestellten Informationen bleibt der Zuschauer am Ende jedoch ratlos zurück. Gut gemeint ist leider eben nicht immer auch gut gemacht [23].

\section{Kampagnen}

Die negativen Effekte von öffentlichen Anti-Impf-Kampagnen auf die Impfbereitschaft der Bevölkerung sind in der medizinischen Literatur gut belegt. In England und Wales trat Pertussis wieder auf, nachdem verstärkte Aktivitäten von Impfgegnern zu einem Absinken der Durchimpfungsrate für DTPw von 81 auf 31 \% geführt hatten [24]. In wenigen Jahren stieg die Inzidenz von Pertussis von unter 10/100 000 Fällen auf über 100/100 000. Erst als diese Entwicklung von der Öffentlichkeit als erneute Bedrohung wahrgenommen wurde, stieg die Akzeptanz der Impfung rapide an. Trotzdem dauerte es fast 10 Jahre, bis Pertussis in England und Wales wieder gut kontrolliert war. 

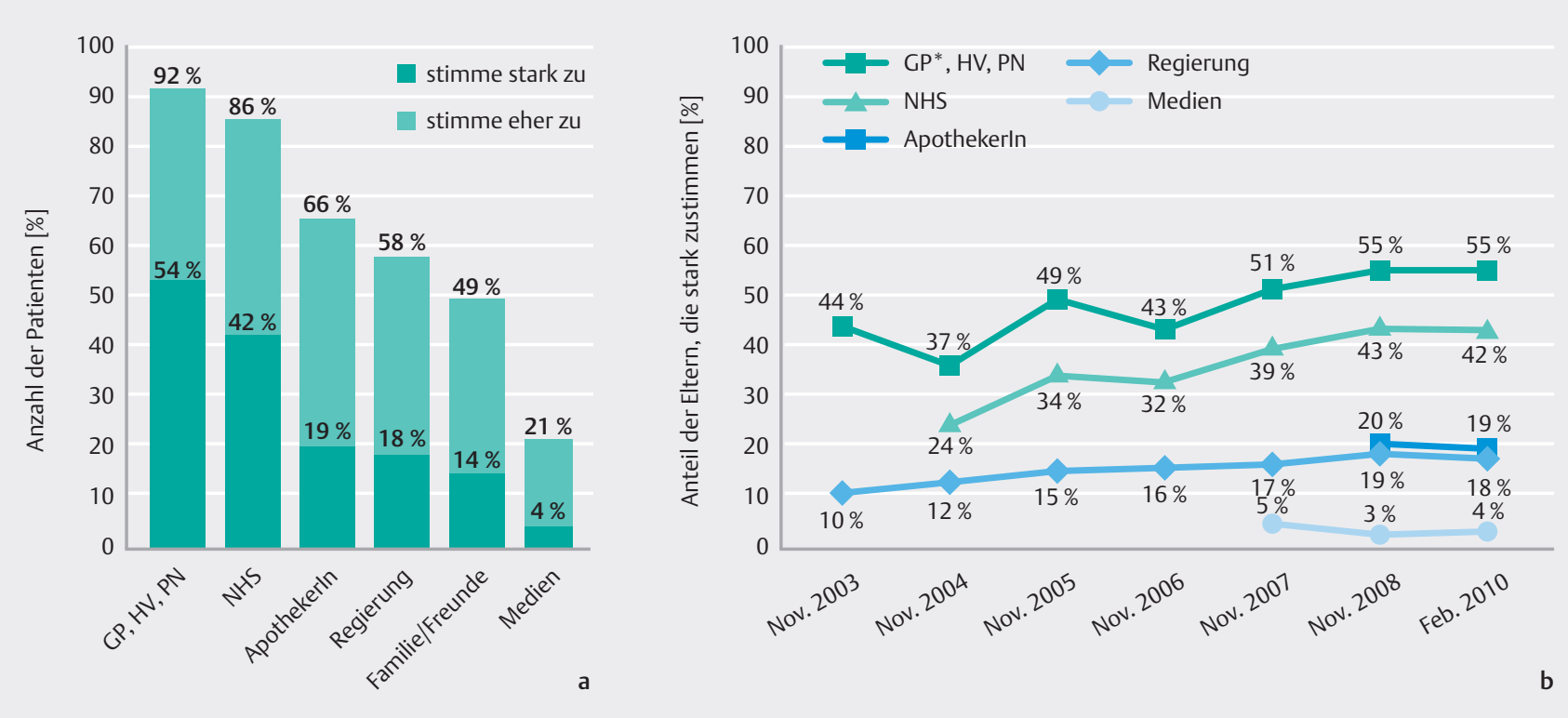

- Abb. 2 Vertrauen in medizinische Informationsquellen. Daten nach [27]. a Wem Eltern bei der Impfberatung vertrauen (2010). Eltern von Kindern im Alter von 0 - 4 Jahren $(n=1730)$. b Wem Eltern bei der Impfberatung vertrauen. Eltern von Kindern im Alter von $0-2$ Jahren $(n=1142)$. GP: general practitioner (Hausarzt), HV: Health Visitor, PN: Practice Nurse (Arzthelferin), NHS: National Health Service. ${ }^{*}$ Daten zu GP vor 2007 erhoben. Daten aus [27].

\section{Internet}

Wenn Eltern heute versuchen, sich über die Risiken und Vorteile von Impfstoffen im Internet zu informieren, sind sie einer Vielzahl von teilweise verwirrenden, nicht immer seriösen Artikeln ausgeliefert. Das Internet ist voll von Blogs und Webseiten, die über Gefahren von Impfstoffen berichten und Eltern mit gemischten Gefühlen hinterlassen, unsicher, welchen Quellen sie vertrauen dürfen. Hier fehlt eine Instanz, die dargebotene Beiträge auf inhaltliche Qualität und Seriosität überprüft und für Laien bewertet. Zudem haben Impfgegner durch das Internet eine neue Dimension der globalen Reichweite und aktiven Einflussnahme erreicht. Im Internet und in sozialen Netzwerken können sich Impfgegner schnell organisieren und ungehindert ohne Qualitätskontrolle ihre kruden Thesen verbreiten.

\section{Merke}

So werden Menschen beeinflusst, die primär nicht unbedingt gegen Impfstoffe eingestellt sind und einfach nur Informationen zu Impfstoffen, Impfstoffsicherheit, Impfplänen und aktuellen Richtlinien suchen.

Eine Studie untersuchte die Bedeutung des Internets für Impfentscheidungen und medizinische Aufklärung in modernen Gesellschaften [25]. Sie bestätigt: Individuelle und öffentliche Bedenken im Zusammenhang mit Impfungen haben das Potenzial, sich über Internet und soziale Medien weltweit schnell und effizient zu verbreiten. Die Autoren weisen jedoch darauf hin, dass auch Gesundheitsbehörden das Internet gezielt für Aufklärungskampagnen zur Förderung des Impfwesens nutzen könnten.
Eine weitere Studie unterstreicht die Sensitivität der Bevölkerung für Informationssuche und Informationsverbreitung über das Internet [26]: Die Veröffentlichung von Fehlinformationen über Nebenwirkungen von Thiomersal (einem heute nicht mehr in Impfstoffen verwendeten Konservierungsstoff) führte innerhalb kürzester Zeit zu einem explosionsartigen Anstieg entsprechender Suchanfragen und Medienberichte im Internet. Richtig genutzt, kann das Internet ganz offensichtlich also auch ein wertvolles Medium zur Förderung des Impfgedankens werden.

\section{Handlungsoptionen}

Um das Verständnis für Impfstoffe und die Akzeptanz von Impfprogrammen zu fördern, müssen Strategien entwickelt werden, die die o. a. Risikowahrnehmungen und komplexen Interaktionen auf dem Weg zur Impfentscheidung berücksichtigen. Interventionen zur Bekämpfung von Impfstoffskepsis haben das Ziel,

- Informationsdefizite aufzulösen und

- das Vertrauen in Impfstoffe, die medizinischen Versorgungsstrukturen und das Gesundheitssystem generell zu stärken.

Dazu gehört auch die Vermittlung von Wissen über Risiken und Komplikationen der durch Impfung vermeidbaren Krankheiten. 
Merke

In diesem Kontext ist es entscheidend, Aufklärungsprogramme zu entwickeln, die eine Kommunikation auf der Basis evidenzbasierter Informationen zur Sicherheit und zum Risiko-Nutzen-Verhältnis von Impfungen ermöglichen [27].

Um das Vertrauen in den Nutzen von Impfungen wiederherzustellen und zu erhalten, sollten die Aktiven und Experten im Gesundheitswesen für Transparenz sorgen: Informationen über Unsicherheiten und mögliche Risiken müssen kommentiert zur Verfügung stehen.

\section{Vertrauen stärken}

Menschen treffen Impfentscheidungen aber nicht nur stringent auf der Basis ihres Wissens über Risiken und Nutzen von Impfstoffen. Der Erfolg wissensbasierter Strategien hängt wesentlich vom Vertrauen ab, das der informierenden Quelle entgegengebracht wird. Vertrauen entsteht durch Dialog und Austausch von Informationen und Meinungen. Eine Vielzahl von Untersuchungen belegt, dass medizinische Anbieter eine entscheidende Rolle bei der Impfentscheidung der Eltern spielen und eine vertrauenswürdige Quelle für Informationen über Impfstoffe sind.

Das britische Gesundheitsministerium überwacht beispielsweise nicht nur die öffentliche Wahrnehmung von Impfstoffen, sondern auch die Frage, welchen Vertretern des Gesundheitswesens die Öffentlichkeit das stärkste Vertrauen entgegenbringt ( $\triangleright$ Abb. 2). Die Daten belegen: Um Impfakzeptanz von Eltern zu erreichen, ist der persönliche Kontakt mit einem informierten und fürsorgenden Kinder- oder Familienarzt der wichtigste Faktor. Deshalb sollten Ärzte, Praxismitarbeiter und andere Ansprechpartner der medizinischen Grundversorgung ihre Anstrengungen um sichere Aufklärung intensivieren und aktiv Missverständnisse ansprechen, die das Vertrauen in Impfungen beschädigen.

\section{KERNAUSSAGEN}

- Impfbedenken sind nicht neu, jedoch hat sich die Wahrnehmung impfbezogener Risiken in den letzten Jahrzehnten gewandelt.

- Neben Aspekten wie Wissen über Impfstoffe, Vertrauen in medizinische Versorgungsstrukturen und politische Institutionen spielen sozio-ökonomische, kulturelle und auch religiöse Standpunkte eine wichtige Rolle. Diese Faktoren unterscheiden sich weltweit und regional erheblich.

- In nahezu allen Erhebungen wird das Vertrauen in Impfstoffe und/oder das Gesundheitssystem als essenziell für eine positive Impfentscheidung identifiziert.

- Das Vertrauen in Impfungen korreliert mit der Einschätzung des speziellen individuellen Erkran- kungsrisikos und der potenziellen Nebenwirkungen der Impfstoffe.

- Die subjektive Risikowahrnehmung entspricht selten den objektiv messbaren Fakten. Sie wird vielmehr durch die individuelle Wahrnehmung in beide Richtungen (Unter- und Überschätzung) verzerrt.

- Eine transparente, wissenschaftsbasierte Kommunikation ist hilfreich, um Vertrauen in das Gesundheitswesen zu fördern und zu erhalten.

- Kinder- und Familienärzte sind für Eltern die wichtigsten Vertrauenspersonen bei Fragen zu Gesundheitsvorsorge und Impfempfehlungen.

Interessenkonflikt

Die Autoren geben an, dass kein Interessenkonflikt besteht.

Autorinnen/Autoren

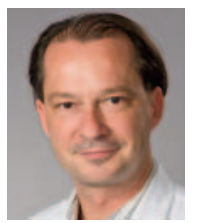

\section{Dr. med. Frank Kowalzik}

Geschäftsführender Oberarzt des Zentrums für Kinder- und Jugendmedizin, Universitätsmedizin Mainz. Schwerpunkte der wissenschaftlichen Arbeit: Infektionsepidemiologie, pädiatrische Immunologie.

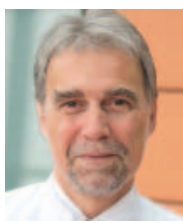

\section{Univ.-Prof. Dr. med. Fred Zepp}

Direktor des Zentrums für Kinder- und Jugendmedizin der Universitätsmedizin Mainz. Facharzt für Kinder- und Jugendmedizin, Neonatologie, pädiatrische Rheumatologie. Wissenschaftliche Schwerpunkte: pädiatrische Immunologie und Infektiologie, Impfstoff-Forschung. Beratende Tätigkeit für öffentliche Gremien, u. a. STIKO.

Korrespondenzadresse

Prof. Dr. Fred Zepp

Zentrum für Kinder- und Jugendmedizin

Universitätsmedizin Mainz

Johannes Gutenberg-Universität

Langenbeckstraße 1

55131 Mainz

fred.zepp@unimedizin-mainz.de

Literatur

[1] Glanz JM, McClure DL, Magid DJ et al. Parental refusal of pertussis vaccination is associated with an increased risk of pertussis infection in children. Pediatrics 2009; 123 (6): 1446 - 1451. doi:10.1542/peds.2008-2150

[2] Glanz JM, Narwaney KJ, Newcomer SR et al. Association between undervaccination with diphtheria, tetanus toxoids, and acellular pertussis (DTaP) vaccine and risk of pertussis infection in children 3 to 36 months of age. JAMA Pediatr 2013; 167: $1060-1064$ 
[3] Poland GA, Jacobson RM. The age-old struggle against the antivaccinationists. N Engl J Med 2011; 364 (2): 97 - 99. doi:10.1056/NEJMp1010594

[4] Larson HJ, Schulz WS, Tucker JD et al. Measuring vaccine confidence: introducing a global vaccine confidence index. PLoS Curr 2015. doi:10.1371/currents.outbreaks. ce0f6177bc97332602a8e3fe7d7f7cc4

[5] Larson H], de Figueiredo A, Xiahong Z et al. The State of Vaccine Confidence 2016: Global Insights Through a 67-Country Survey. EBioMedicine 2016; 12: 295-301. doi:10.1016/j. ebiom.2016.08.042

[6] Rey D, Fressard L, Cortaredona S et al. On Behalf Of The Baromètre Santé Group. Vaccine hesitancy in the French population in 2016, and its association with vaccine uptake and perceived vaccine risk-benefit balance. Euro Surveill 2018; 23 (17). doi:10.2807/1560-7917.ES.2018.23.17.17-00816

[7] Baumgaertner B, Carlisle JE, Justwan F. The influence of political ideology and trust on willingness to vaccinate. PLoS One 2018; 13 (1): e0191728. doi:10.1371/journal.pone.0191728

[8] Danis K, Georgakopoulou T, Stavrou T et al. Socio-economic factors play a more important role in childhood vaccination coverage than parental perceptions: a cross-sectional study in Greece. Vaccine 2010; 28 (7): 1861 - 1869

[9] Babalola S. Maternal reasons for non-immunization and partial immunization in northern Nigeria. J Paediatr Child Health 2011; 47 (5): $276-281$

[10] Brown K, Fraser G, Ramsay M et al. Attitudinal and demographic predictors of measles-mumps-rubella vaccine [MMR] uptake during the UK catch-up campaign 2008-2009: crosssectional survey. PLoS ONE 2011: e19381. doi:10.1371/journal. pone. 0019381

[11] Falagas ME, Zarkadoulia E. Factors associated with suboptimal compliance to vaccinations in children in developed countries: a systematic review. Curr Med Res Opin 2008; 24 (6): 17191741

[12] Rainey J], Watkins M, Ryman TK et al. Reasons related to nonvaccination and under-vaccination of children in low and middle income countries: findings from a systematic review of the published literature 1999-2009. Vaccine 2011; 29 (46): $8215-8221$

[13] Prematunge C, Corace K, McCarthy A et al. Factors influencing pandemic influenza vaccination of healthcare workers - a systematic review. Vaccine 2012; 30 (32): $4733-4743$

[14] Trim K, Nagji N, Elit L et al. Parental knowledge, attitudes, and behaviours towards human papillomavirus vaccination for their children: a systematic review from 2001 to 2011. Obstet Gynecol Int 2012; 2012: 921236. doi:10.1155/2012/921236

[15] Mills EJ, Montori VM, Ross CP et al. Systematically reviewing qualitative studies complements survey design: an exploratory study of barriers to paediatric immunizations. J Clin Epidemiol 2005; 58 (11): $1101-1108$
[16] Betsch C, Schmid P, Heinemeier D et al. Beyond confidence: Development of a measure assessing the $5 \mathrm{C}$ psychological antecedents of vaccination. Preprint Psyarxiv, last edited August 11, 2018. doi:10.31234/osf.io/ytb7w

[17] McClure CC, Cataldi JR, O'Leary ST. Vaccine Hesitancy: Where We Are and Where We Are Going. Clinical Therapeutics 2017; 39 (8): 1550 - 1562. doi:10.1016/j.clinthera.2017.07.003

[18] Schwermer B. Objektives Gripperisiko, subjektive Risikowahrnehmung und Impfbereitschaft: Wie reagiert die Bevölkerung auf Gesundheitskommunikation? Das Gesundheitswesen 2013; 75 (5): e23 - e27. doi:10.1055/s-0032-1331789

[19] Renner B, Schupp HS, Schmälzle R. Risikowahrnehmung und Risikokommunikation. In: Bengel J, et al. Hrsg. Handbuch der Gesundheitspsychologie und Medizinischen Psychologie. Göttingen [u. a.]: Hogrefe; 2009: 113-121

[20] Lichtenstein S, Slovic P, Fischhoff B et al. Judged frequency of lethal events. Journal of Experimental Psychology: Human Learning and Memory 1978; 4: 551-578

[21] Brewer NT, Chapman GB, Gibbons FX et al. Meta-analysis of the relationship between risk perception and health behavior: the example of vaccination. Health Psychol 2007; 26 (2): $136-145$

[22] Gerber JS, Offit PA. Vaccines and autism: A tale of shifting hypotheses. Clin Infect Dis 2009; 48 (4): 456-461

[23] Dokumentation „Eingeimpft“ und eine Art wissenschaftliche Rezension. Science Media Center Germany. 17.08.2018. Im Internet: www.sciencemediacenter.de/alle-angebote/rapidreaction/details/news/dokumentation-eingeimpft-und-eineart-wissenschaftliche-rezension

[24] Gangarosa E], Galazka AM, Wolfe CR et al. Impact of anti-vaccine movements on pertussis control: the untold story. Lancet 1998; 351: (9099) 356-361

[25] Betsch C, Brewer NT, Brocard P et al. Opportunities and challenges of Web 2.0 for vaccination decisions. Vaccine 2012; 30 (25): $3727-3733$

[26] Myers MG, Pineda D. Misinformation about Vaccines. In: Barrett ADT, Stanberry LR, eds.; Vaccines for Biodefense and Emerging and Neglected Diseases. Oxford: Academic Press; 2009: $256-271$

[27] Larson H], Cooper LZ, Eskola J et al. Addressing the vaccine confidence gap. Lancet 2011; 378: 526-535

Bibliografie

DOI https://doi.org/10.1055/a-0479-3756

Dtsch Med Wochenschr 2019; 144: 254-261

(c) Georg Thieme Verlag KG, Stuttgart · New York ISSN 0012-0472 